\title{
Noninvasive Brain Stimulation Using a Modulated Microwave Signal
}

\author{
Taeyoon $\mathrm{Seo}^{1} \cdot$ Seongwoog $\mathrm{Oh}^{2} \cdot$ Dahee Jung, $2,3,5 \cdot$ Yeowool Huh ${ }^{2,3}$. \\ Jeiwon $\mathrm{Cho}^{2,3} \cdot$ Youngwoo Kwon ${ }^{1,}$
}

\begin{abstract}
We propose a microwave signal generation system for brain stimulation. The existing brain stimulation system uses a signal of several tens of $\mathrm{kHz}$, and the magnetic field distribution is wide. Microwave is used to locally limit the distribution of the electromagnetic field and to change the action potential of the cell with less power. The switch modulates the microwave signal to obtain a pulse envelope. The action potential of the cell can be controlled to the excitation/inhibition state by adjusting the repetition frequency. These results are confirmed by measuring the cell potential of the mouse brain.
\end{abstract}

Key Words: Microwave Brain Stimulation, Noninvasive Brain Stimulation, Pulse Modulated Stimulation Signal.

\section{INTRODUCTION}

The experience of a new environment can lead to changes in the connections of the nerves throughout life [1]. The ability of the brain to reorganize itself by forming new neural connections is called neuroplasticity [2]. Neuroplasticity is the basic mechanism of learning and memory and is the result of the restoration of functions after cerebral nerve damage. Conventional rehabilitation methods do not directly change the brain but mostly improve the function of the brain through appropriate environmental changes.

Noninvasive brain stimulation is a method of neuromodulation by stimulating certain parts of the brain without surgical treatment using a magnetic field or an electric current. A transcranial magnetic stimulation (TMS) is currently being used clinically [3]. Although TMS has the advantages of noninvasiveness and inability to cause skin irritation, it has the disadvantages of requiring expensive equipment and causing noise. TMS is based on the induction current induced by a change in magnetic field. The magnetic field in the tens of $\mathrm{kHz}$ is widely distributed to include all areas of the brain. Localizing the stimulation area in the brain is possible because microwave has a short skin depth.

In this study, microwave is used as a method to change the action potential of the nerve. Moreover, the nerve firing rate (FR) is modulated by controlling the pulse repetition frequency (PRF).

\section{INTEGRATED BRAIN STIMULATION SYSTEM}

Manuscript received September 27, 2017 ; Revised November 15, 2017 ; Accepted November 30, 2017. (ID No. 20170927-056J)

${ }^{1}$ Department of Electrical and Computer Engineering, Institute of New Media and Communications, Seoul National University, Seoul, Korea.

${ }^{2}$ Department of Medical Science, Catholic Kwandong University International St. Mary's Hospital, Incheon, Korea.

${ }^{3}$ Institute for Bio-Medical Convergence, Incheon St. Mary's Hospital, College of Medicine, The Catholic University of Korea, Incheon, Korea.

${ }^{4}$ Department of Neuroscience, Korea University of Science and Technology, Seoul, Korea.

${ }^{5}$ Center for Neuroscience, Korea Institute of Science and Technology, Seoul, Korea.

"Corresponding Author: Youngwoo Kwon (e-mail: ykwon@snu.ac.kr)

This is an Open-Access article distributed under the terms of the Creative Commons Attribution Non-Commercial License (http://creativecommons.org/licenses/by-nc/4.0) which permits unrestricted non-commercial use, distribution, and reproduction in any medium, provided the original work is properly cited.

(c) Copyright The Korean Institute of Electromagnetic Engineering and Science. All Rights Reserved. 
To demonstrate the feasibility of brain stimulation using microwave, an integrated system is implemented by combining a voltage-controlled oscillator (VCO), switches, and a power amplifier (PA). The stimulus signal is transmitted to the brain through a stimulator. The block diagram of the system is shown in Fig. 1.

\section{Voltage-Controlled Oscillator}

As a microwave signal source, a $\mathrm{VCO}$ generates a $6.5-\mathrm{GHz}$ microwave signal. The schematic of the VCO is shown in Fig. 2. The VCO is designed with a cross-coupled structure, which is widely adopted in VCO design. A buffer isolates the load impedance effects and offers voltage gain. By varying the load impedance of the common-source amplifier, the buffer controls the output power of the stimulation system.

\section{Power Amplifier}

As shown in Fig. 3, shunt switches connected with the buffer act as a modulator. A stimulation protocol is applied to the gate of the switch transistor, so that the envelope of the microwave signal has a pulse shape.

The PA amplifies the modulated microwave signal. Given the output power of the buffer (around $0 \mathrm{dBm}$ ), a two-stage amplifier is designed for high power $(>20 \mathrm{dBm})$. A differential structure is introduced to avoid the source degeneration effect.

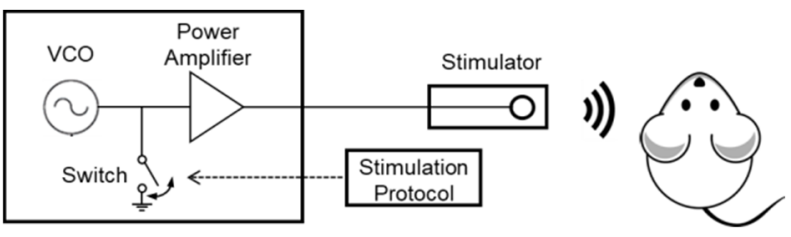

Fig. 1. Overall block diagram of the proposed brain stimulation system.

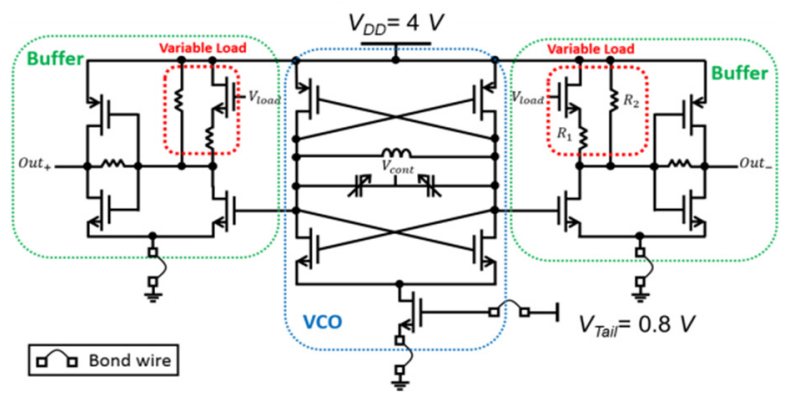

Fig. 2. A schematic of the designed VCO and variable load.

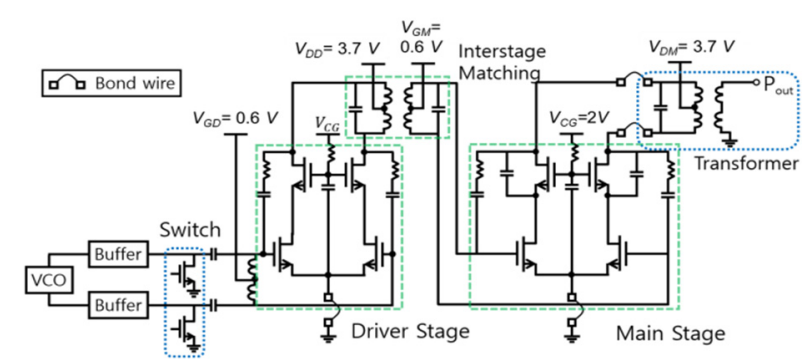

Fig. 3. A schematic of the designed switch and PA.

\section{Stimulator}

The stimulator is fabricated using two 0.254 -mm-thick substrates, RO5880, with $\varepsilon_{r}$ of 2.2. An aperture is the cross-section of the coaxial cable [4]. The structure and the photograph of the stimulator are shown in Fig. 4. Similar to the characteristics of an open-ended coaxial cable, the electric field is distributed on the surface of the aperture. Therefore, it affects only the action potential of the brain surface cell and not the deep brain.

\section{Integrated Brain Stimulation System Using a Modulated Mi- crowave Signal}

The simulation system is implemented by integrating all of the circuits above. All of the active circuit components (VCO, switches, and PA) are fabricated using the $0.28 \mu \mathrm{m}$ SOI CMOS process. The bias voltage is given in Figs. 2 and 3. Fig. 5 shows the photographs of the proposed system. In Fig. 5(b), a transformer converts the differential to a single-ended structure, which is the stimulator. To minimize combining loss, a sevenlayer printed circuit board is used to fabricate the transformer.

Fig. 6(a) and (b) show the measured characteristics of the system. The frequency tuning range covers $5.7-7.12 \mathrm{GHz}$, and the output power varies at $3.2-22.4 \mathrm{dBm}$ at $6.5 \mathrm{GHz}$. The measured $\left|S_{11}\right|$ of the stimulator in contact with mouse brain is 6.1 $\mathrm{dB}$ at $6.5 \mathrm{GHz}$ as plotted in Fig. 6(c). Fig. 6(d) presents the pulse modulated stimulation signal at the output of the switch. The current consumptions of the $\mathrm{VCO}$ and PA are $64 \mathrm{~mA}$ and $180 \mathrm{~mA}$, respectively.

\section{EXPERIMENTAL RESULTS}

A mouse is used to determine the feasibility of the modulated microwave stimulation. The mouse is anesthetized, and the skin and skull are incised. Experimental setup is shown in Fig. 7(a).
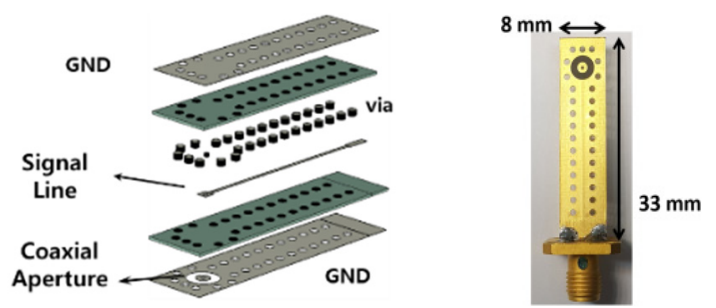

Fig. 4. Stimulator fabricated using double-layer printed circuit board technology.

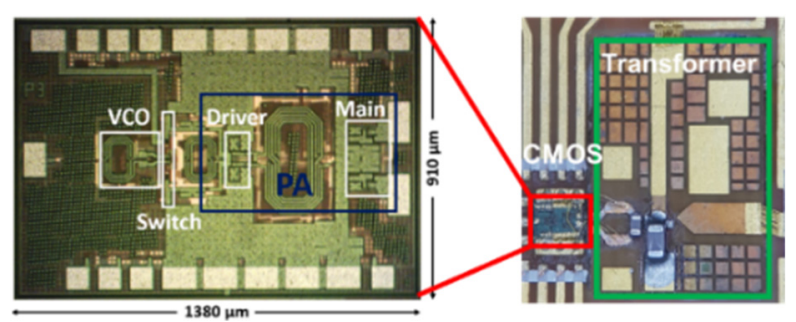

Fig. 5. Photograph of the integrated system. 


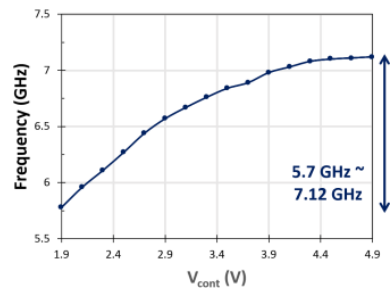

(a)

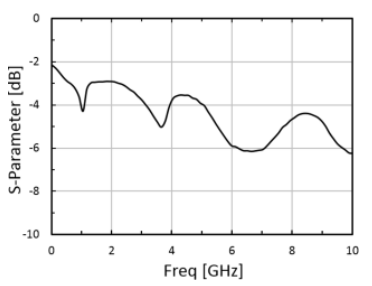

(c)

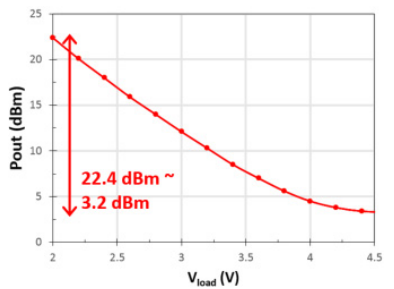

(b)

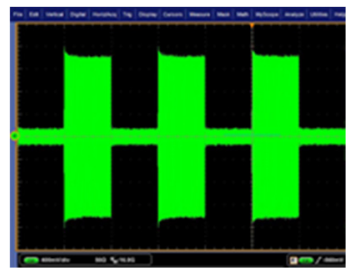

(d)
Fig. 6. Measurement results of the system: (a) frequency tuning range, (b) output power, (c) $\left|S_{11}\right|$ of the designed stimulator, and (d) pulse modulated output signal.

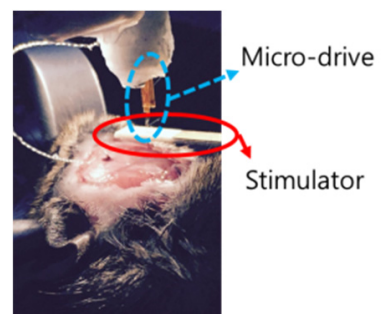

(a)

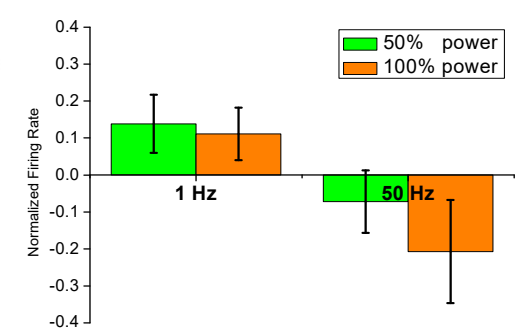

(b)
Fig. 7. (a) Experimental setup for mouse brain stimulation. (b) Stimulation results normalized according to the baseline.

The aperture of the stimulator is brought into direct contact with the brain. Then, a micro-drive consisting of four bundles with four nichrome wires is inserted, targeting the hippocampus of the brain. The brain signals are filtered $(600 \mathrm{~Hz}-6 \mathrm{kHz})$ at a sampling rate of $30,303 \mathrm{~Hz}$ through a data acquisition system (Digital Lynx 4SX; Neuralynx, Bozeman, MT, USA) and sorted into single-unit cell data. The effect of the modulated microwave signal on the brain stimulation is observed by comparing the FR of the single cell data before and after the stimulation. The FR of single cell data before stimulation is used as the baseline.

After the baseline measurement, the cell is stimulated with $50 \%$ power $(2 \mathrm{~mW})$ for 5 minutes. The pulse of the stimulation signal is $1 \mathrm{~Hz}$ at $1 \%$ duty cycle (10 ms width). The observation time for 5 minutes is then secured. The observation follows the same protocol as described above at $100 \%$ power $(4 \mathrm{~mW})$. The mouse is allowed to rest for 1 hour and is then given the same stimulus to the other cell. The $1 \mathrm{~Hz}$ stimulation is measured in six cells. The $50-\mathrm{Hz}$ stimulation signal has a $50 \%$ duty cycle $(10$ ms pulse width). It is analyzed for the activity of seven cells using the same protocol as above.

For the neuronal activity analysis, the FR changes of individual neurons through stimulation are calculated using the fol- lowing equation:

$$
\begin{aligned}
& \text { Normalized firing rate }(\mathrm{Hz})= \\
& \frac{\text { After stimulation-Before stimulation }}{\text { After stimulation }+ \text { Before stimulation }} .
\end{aligned}
$$

Therefore, values greater than 0 indicate increased activity compared with the baseline, and small values indicate a decreased activity. The range bars indicate the standard deviations. In the $1 \mathrm{~Hz}$ case, the FR is excited and inversely inhibited at 50 $\mathrm{Hz}$ as in Fig. 7(b).

\section{CONCLUSION}

We have demonstrated a microwave brain stimulation using pulse modulation to enhance/inhibit the FR of a cell in the brain. The system consists of a VCO, switches, a PA, and a stimulator. This system configuration has the advantage of easily adjusting the pulse width and the PRF. The stimulation effect is confirmed by measuring the action potential of the mouse brain. The opposite cell activity is obtained according to the PRF of the modulation.

Microwave brain stimulation is advantageous in terms of energy consumption and system size compared with the conventional TMS. This study requires in-depth research of stimulation signal variations and is expected to lead to the development of neuroscience research.

This research was supported by Basic Science Research Program through the National Research Foundation of Korea funded by the Ministry of Science, ICT, and Future Planning (No. NRF-2015R1A2A2A04005487), Brain Science Research Program (No. 2015M3C7A1028392 and No. 2015M3C7A1028393), and Brain Korea 21 Plus Project in 2017.

\section{REFERENCES}

[1] D. O. Hebb, "The effects of early experience on problem solving at maturity," American Psychologist, vol. 2, pp. 306307, 1947.

[2] B. H. Dobkin, The Clinical Science of Neurological Rehabilitation, 2nd ed. New York, NY: Oxford University Press, 2003.

[3] S. Rossi, M. Hellett, P. M. Rossini, A. Pascual-Leone, and The Society of TMS Consensus Group, "Safety, ethical considerations, and application guidelines for the use of transcranial magnetic stimulation in clinical practice and research," Clinical Neurophysiology, vol. 120, no. 12, pp. 20082039, 2009.

[4] Y. Kwon, K. Kim, S. H. Hwang, T. Seo, and Y. K. Kim, "Active integrated probes for tumor detection and ablation," in Proceedings of 2014 IEEE MTT-S International Microwave Symposium (IMS), Tampa, FL, 2014, pp. 1-4. 